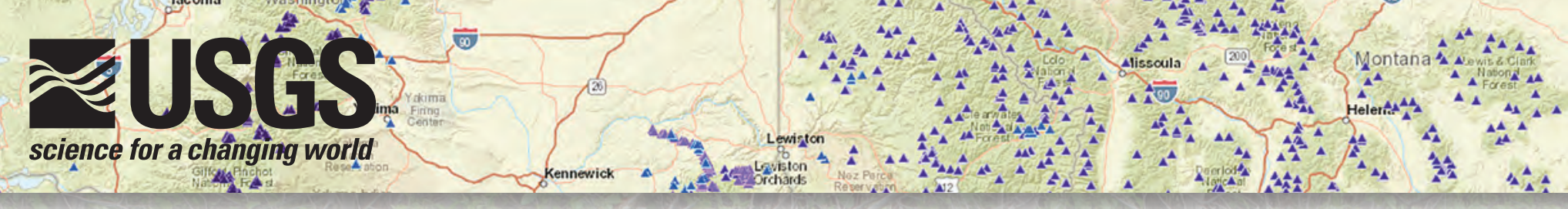

\title{
MonitoringResources.org-Supporting Coordinated and Cost-Effective Natural Resource Monitoring Across Organizations
}

\begin{abstract}
Natural resource managers who oversee the Nation's resources require data to support informed decision-making at a variety of spatial and temporal scales that often cross typical jurisdictional boundaries such as states, agency regions, and watersheds. These data come from multiple agencies, programs, and sources, often with their own methods and standards for data collection and organization. Coordinating standards and methods is often prohibitively time-intensive and expensive. MonitoringResources.org offers a suite of tools and resources that support coordination of monitoring efforts, cost-effective planning, and sharing of knowledge among organizations. The website was developed by the Pacific Northwest Aquatic Monitoring Partnership a collaboration of Federal, state, tribal, local, and private monitoring programs-and the U.S. Geological Survey (USGS), with funding from the Bonneville Power Administration and USGS. It is a key component of a coordinated monitoring and information network.
\end{abstract}

\section{What is MonitoringResources.org?}

MonitoringResources.org provides an online, publicly accessible suite of information and tools for natural resource monitoring programs and professionals. Tools and information include maps and metadata describing existing monitoring projects; a library of standard monitoring methods and protocols; and guidelines and tools to help managers develop new monitoring programs and share existing ones. Resource managers, funding agencies, and policy makers can quickly look up existing and proposed monitoring projects so that they can better understand how well priorities are being met and assess where monitoring efforts might have gaps or redundancies. Users can see maps of sampling sites, compare protocols of different monitoring projects, and identify locations and sampling strategies for additional monitoring or to optimize existing monitoring. The tools guide and support study design and methods documentation from the earliest planning stages through implementation. Users can easily add information about their projects and programs and can share the information with partners.

\section{Tools for Managing a Project Through the Data Lifecycle}

MonitoringResources.org supports the need to describe and document projects throughout the data management lifecycle by providing a structured, publicly accessible resource for recording project metadata. The site's integrated modules work together and also connect to external systems, such as curated data repositories, GIS databases, and program websites. Monitoring data from programs and projects that use MonitoringResources.org are more valuable because they are accompanied by consistent, persistent, and accessible documentation of users' protocols, methods, and designs.

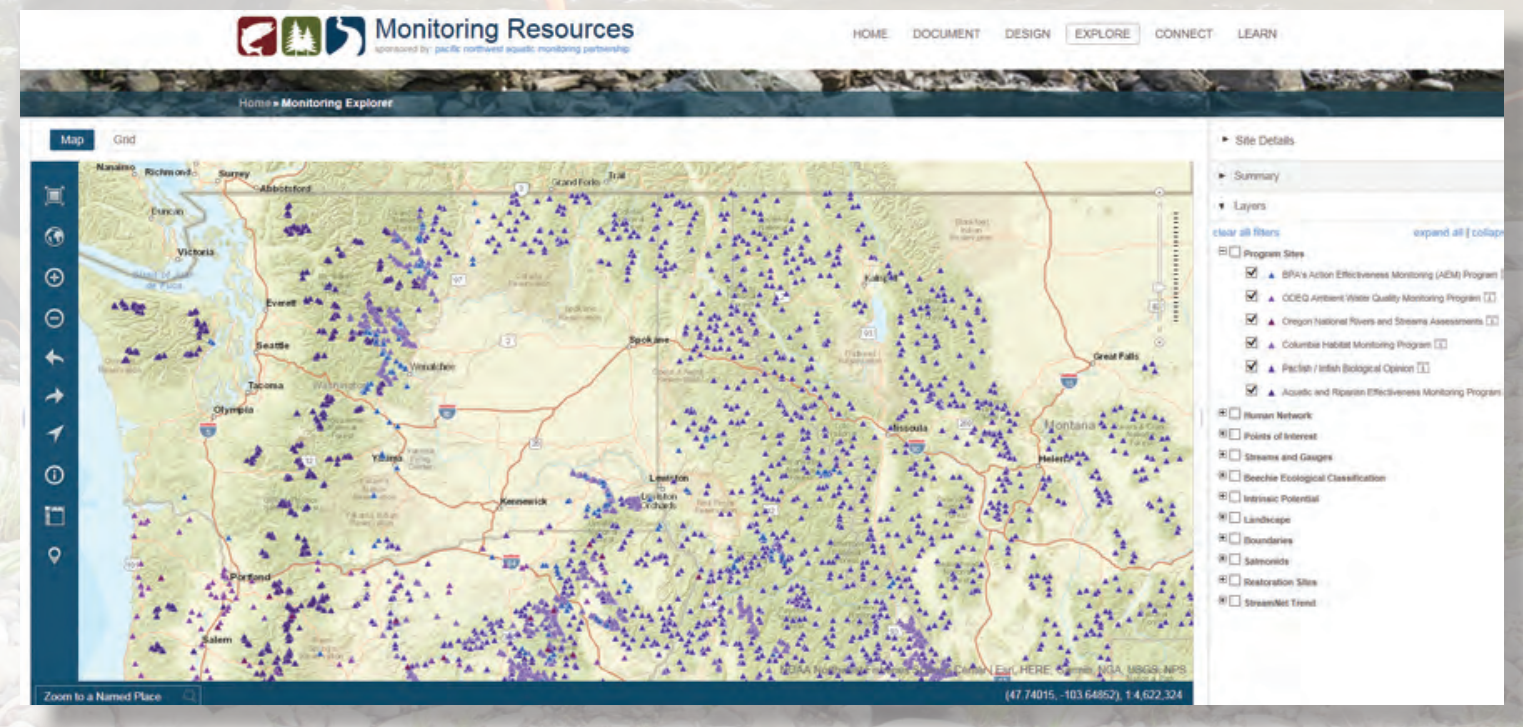

Screen shot of the Monitoring Explorer tool showing program sampling sites from six monitoring and assessment programs operating within the Pacific Northwest. 


\section{Modules Available at MonitoringResources.org}

- Study Design Tool helps define project objectives and an overall monitoring plan.

- Method Builder and Library supports documentation of the step-by-step processes for collecting and analyzing data.

- Protocol Builder and Library allows users to combine multiple methods into a protocol to define the suite of information collected and analyzed, including use of previously published methods from the method library.

- Master Sample Library stores, displays, and documents master samples - a master sample consists of a set of potential sample locations from which monitoring sites can be selected. Use of master samples allows data from multiple monitoring programs to be more easily shared, integrated, and used for other applications.

- User Sample File leads users through the process of uploading planned sampling sites to MonitoringResources.org.

- Sample Designer Tool helps users

- Create and document multiple types of sample designs;

- Use a master sample to select and document spatially balanced, random sample sites and define the temporal plan for sampling; the selection process uses the Generalized Random Tessellation Stratified (GRTS) algorithm commonly used by agencies, monitoring organizations, and scientists;

- Track site evaluation status before and during sampling; and

- Share sampling dates, times, and implementation details with others.

- Monitoring Explorer provides an interactive map viewer that displays where, when, and how various projects have collected data. Users can search for and display sample sites and their associated metadata.

- Application Programming Interfaces (APIs) enable automated sharing of a user's MonitoringResources.org content with other programs, projects, or databases.

\section{Tools for Communication and Collaboration}

MonitoringResources.org helps users connect and collaborate with other natural resource managers and scientists. The website provides educational materials, a community forum, a glossary, and links to environmental information repositories where monitoring data are stored and shared. These tools help users discover the "who, what, when, where, and how" of monitoring activities. Within a single project, users can share protocols and standardize site selection methodologies, data collection efforts, and data analyses. Managers and scientists working on different projects can learn about previous monitoring efforts and can coordinate data collection for cross-site comparisons.

\section{Organizations Already Using MonitoringResources.org}

The Bonneville Power Administration (BPA) invested in MonitoringResources.org to help coordinate and standardize protocols for the monitoring projects it had funded in the Pacific Northwest. Starting in 2017, interest from users outside of the Northwest has resulted in new work to expand the geographic scope of the tool. Organizations and projects that rely on the recently expanded toolset include

- BPA Fish and Wildlife Program, for documentation of protocols, designs, and sites for BPA-funded research, monitoring, and evaluation of projects throughout the Columbia River Basin;

- Columbia Habitat Monitoring Program, for design and protocol documentation to support multiple entities participating in a Columbia River Basin-wide program;

- Bureau of Land Management Assessment, Inventory, and Monitoring Program, to determine and document aquatic sampling sites across 13 western states; and

- North American Bat Monitoring Program, to design, document, and display sites for multi-partner coordination of continental-scale bat population monitoring.

\section{Benefits of Using MonitoringResources.org}

MonitoringResources.org adds significant value to monitoring programs by enhancing monitoring quality, planning, coordination, efficiency, and cost-savings. The site

- Supports efficient use of funding by supporting collaboration and coordination of monitoring efforts;

- Provides information about current and pending multi-jurisdictional monitoring activities so that gaps, redundancies, and opportunities for collaboration can be considered;

- Provides clear, consistent documentation of data collection and analysis methods;

- Helps managers find data and information to support synthesis of existing data;

- Informs future use of data by providing persistent documentation of how data were collected; and

- Promotes transfer of knowledge among monitoring practitioners and program managers.

For more information contact:

Jennifer M. Bayer

U.S. Geological Survey, 5501A Cook-Underwood Road

Cook, WA 98605

jbayer@usgs.gov; (503) 201-4179

To learn more, visit www.MonitoringResources.org. 